\title{
A Fundamental Wave Amplitude Prediction Algorithm Based on Fuzzy Neural Network for Harmonic Elimination of Electric Arc Furnace Current
}

\author{
Wanjun Lei, Yanxia Wang, Lu Wang, and Hui Cao \\ State Key Laboratory of Electrical Insulation and Power Equipment, School of Electrical Engineering, Xian Jiaotong University, Xian, \\ Shaanxi 710049, China \\ Correspondence should be addressed to Hui Cao; huicao@mail.xjtu.edu.cn
}

Received 11 April 2015; Accepted 25 May 2015

Academic Editor: William Guo

Copyright (C) 2015 Wanjun Lei et al. This is an open access article distributed under the Creative Commons Attribution License, which permits unrestricted use, distribution, and reproduction in any medium, provided the original work is properly cited.

\begin{abstract}
Electric arc furnace (EAF) causes the harmonics to impact on the supply network greatly and harmonic elimination is a very important research work for the power quality associated with EAF. In the paper, a fundamental wave amplitude prediction algorithm based on fuzzy neural network for harmonic elimination of EAF current is proposed. The proposed algorithm uses the learning ability of the neural network to refine Takagi-Sugeno type fuzzy rules and the inputs are the average of the current measured value in different time intervals. To verify the effectiveness of the proposed algorithm, some experiments are performed to compare the proposed algorithm with the back-propagation neural networks, and the field data collected at an EAF are used in the experiments. Moreover, the measured amplitudes of fundamental waves of field data are obtained by the sliding-window-based discrete Fourier transform on the field data. The experiments results show that the proposed algorithm has higher precision. The real curves also verify that the amplitude of fundamental wave current could be predicted accurately and the harmonic elimination of EAF would be realized based on the proposed algorithm.
\end{abstract}

\section{Introduction}

Electric arc furnace (EAF) smelts the raw metal by generating arcs and has been widely used in the steel industry. For the characteristics of electric arc and meltdown processes, EAF causes some undesirable disturbing effects, such as harmonics and flicker, which would have high impact on the supply network [1]. Therefore, harmonic elimination is of important theoretical significance and practical motivation for the power quality associated with EAF.

Traditionally, most researches focus on the steady-state modeling for harmonic elimination and some improved models of EAF are presented recently. The cubic spline interpolation is proposed to model the voltage-current characteristic of EAF [2], and a new EAF-specific model based on field measurements of instantaneous furnace voltages and currents is presented [3]. Moreover, a mathematical model of the 3-phase AC of EAF is proposed in a more macroscopic level of modeling [4]. However, due to the instability of smelting process and the randomness of the arc generation, establishing a precise model of the EAF is extremely difficult. Moreover, because some physical responses of EAF cannot be obtained from the theoretical study easily, the assumptions of parameters would simplify the EAF model and the analysis results would be affected. Artificial neural network (ANN) learns the relationship between output and input by adjusting the interconnections of neurons and has been adopted for harmonic elimination [5-11]. In particular, an ANN-based inductances on-line estimation of the swinging power cables is discussed for efficient power control of EAF [12]. Nevertheless, opaqueness is major shortcoming of ANN. Hence, to deal with this problem, the fuzzy neural network (FNN) is proposed [13]. FNN is a combined system with ANN and fuzzy logic technique. The fuzzy concepts could improve the transparency for understanding the inner working of ANN, and for fuzzy logic technique, the fuzzy rules have clear semantic meanings; namely, FNN has the advantages of learning optimization ability of ANN and the human-like thinking of fuzzy logic technique. In addition, the parameter of FNN could be determined easily. Adaptive 


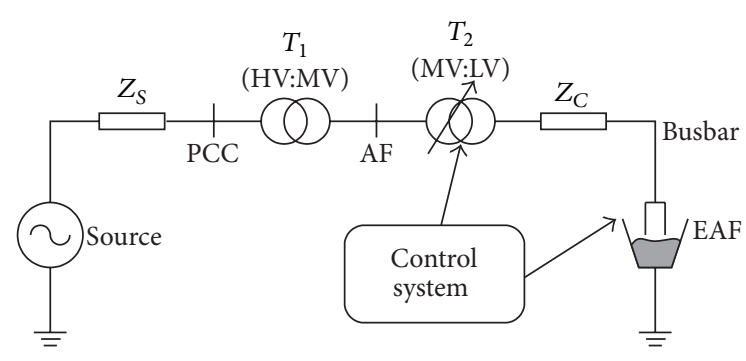

FIGURE 1: Diagram of EAF power supply system model.

neurofuzzy inference system (ANFIS) is one kind of FNN and a first-order Takagi-Sugeno fuzzy system. Since ANFIS could excellently estimate a system with uncertainty, a new method based on ANFIS is proposed to evaluate the slag quality in EAF using power quality indices [14].

This paper proposes a fundamental wave amplitude prediction algorithm based on FNN for harmonic elimination of EAF current. For the proposed algorithm, ANFIS is used to predict the real-time amplitude of fundamental wave current of EAF, which could provide a guarantee for the realization of harmonic elimination. The inputs are the average of the current measured value in different time intervals. To verify the effectiveness of the proposed algorithm, some experiments are performed on the field data collected at an EAF. In the experiments, the proposed algorithm is compared with the back-propagation neural networks (BPNN) for estimating the fundamental wave amplitude. Moreover, the measured values of fundamental wave amplitude of field data are obtained by the sliding-window-based discrete Fourier transform (SW-DFT) on the field data. The paper is organized as follows. The power supply system model and the equivalent circuit of EAF are described in Section 2. In Section 3, the proposed algorithm is presented in detail. In Section 4, the experiments results are discussed to verify the effectiveness of the proposed algorithm. Finally, Section 5 concludes the paper.

\section{Electric Arc Furnace}

The EAF power supply system model is shown in Figure 1. $T_{1}$ is a supply transformer and $T_{2}$ is a furnace transformer. $Z_{S}$ is the grid impedance and $Z_{C}$ is the busbar impedance of EAF. According to the state variables of EAF, such as the arc length, the current value, and the temperature, the controller could regulate the stalls of output voltage of the furnace transformer and the electrode position. The equivalent circuit is shown in Figure 2. $R_{s}$ and $L_{s}$ are equivalent to the grid impedance. The transformer with a ratio of $T_{p}: 1$ is equivalent to $T_{1}$ and $T_{2}$. $R_{c}$ and $L_{c}$ are equivalent to the busbar impedance. $V_{s}$ is the power source. Time-varying resistance $R_{E}$ and $V_{b}$ are the arc equivalent model.

\section{The Proposed Algorithm}

According to the characteristics of EAF, a fundamental wave amplitude prediction algorithm is presented in the paper

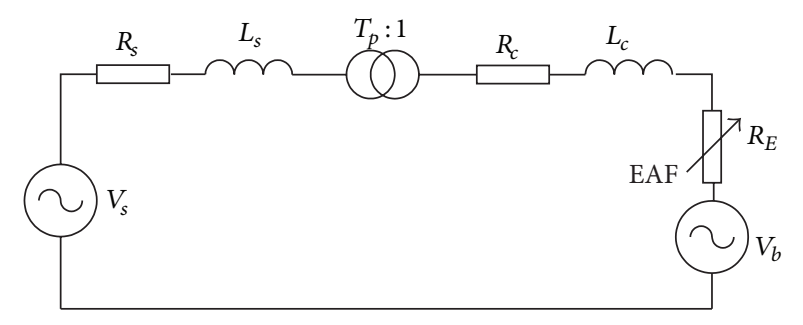

FIgURE 2: Equivalent circuit of EAF.

and the architecture of the proposed algorithm is shown in Figure 3. By predicting the fundamental wave amplitude, the nonfundamental components of EAF current are separated out and used as the command signal to control the power electronic converter to generate the harmonic current, which could counteract the harmonic of EAF, so the grid current only contains the fundamental wave. The output of the proposed algorithm, $i_{f}(t+n+1)$, is the prediction amplitude of fundamental wave current and $n$ is the number of inputs. The inputs of the proposed algorithm are the average of the current measured value in different time intervals; for example, $i_{m}\left(t_{n}\right)$ is the $n$th inputs and could be obtained by the following equation:

$$
i_{m}\left(t_{n}\right)=\frac{1}{k} \sum_{j=0}^{k} i_{m}(t+(n-1)(k+1)+j),
$$

where $k$ is a fixed integer and equals the number of samplings in time intervals.

For the proposed algorithm, ANFIS is adopted for predicting the amplitude of fundamental wave current of EAF, and the architecture is shown in Figure 2. The algorithm has five layers. In the same layer, the nodes are with the same activation function. To facilitate the implementation of the proposed algorithm, each input uses the same linguistic term, and the linguistic terms set is $\left\{A_{1}, A_{2}, \ldots, A_{m}\right\}$, where $m$ is the number of fuzzy linguistic terms. Since the membership functions are determined automatically by training process, the value of $m$ may not be set beforehand. In layer 1 , each node function is the membership values of each input with respect to its linguistic term $A_{L}, L \in\{1,2, \ldots, m\}$, and the Gaussian function is used as the membership functions. In layer 2 , each node is a circle node labeled $\prod$ and plays the role of a simple multiplier. There are $m^{n}$ nodes in this layer and the output of each node represents the firing strength of the rule. In layer 3 , each node is a circle node labeled $N$ and calculates the ratio of the activation level of the $i$ th rule to the total of all activation level. In layer 4 , there are $m^{n}$ nodes in the layer and each node calculates the contribution of the overall output; namely, it is simply the product of the normalized firing strength and the function of consequent, which is a first-order polynomial. Layer 5 has only one node labeled $\sum$ to indicate that it performs the function of a simple summer. The output of the node is final output, $i_{f}(t+n+1)$, which is 


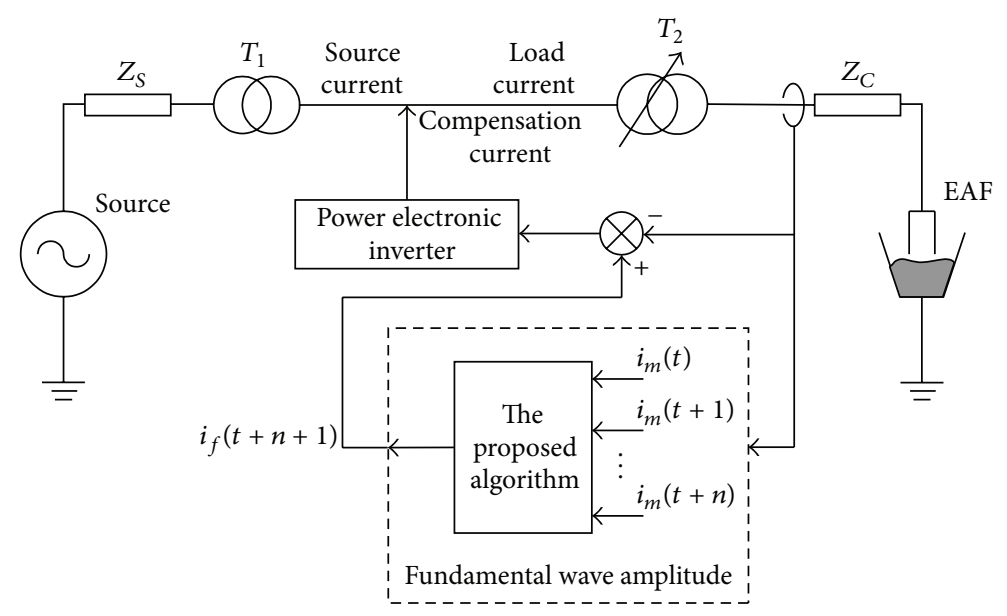

FIGURE 3: The proposed algorithm for harmonic elimination of EAF current.

the prediction amplitude of fundamental wave current and is given by

$$
i_{f}(t+n+1)=\sum_{q=1}^{m^{n}}\left\{\frac{w_{i}}{\sum_{p=1}^{m^{n}} w_{p}} \cdot\left(\sum_{c=1}^{m} a_{c}^{q} x_{c}+b_{q}\right)\right\},
$$

where $a_{c}^{q}$ and $b_{q}$ are the consequent parameters.

For the proposed algorithm, a hybrid learning process is adopted to tune all the modifiable parameters to make the output match the measured value. The hybrid learning process adjusts the consequent parameters in a forward pass and the antecedent parameters in a backward pass [15]. This is a linear combination of the modifiable parameters. For this observation, we can divide the parameter set into two sets. Consider

$$
P=P_{1} \oplus P_{2}
$$

where $P$ is the set of total parameters. $P_{1}$ is the set of antecedent parameters. $P_{2}$ is the set of consequent parameters. $\oplus$ is the sum operation.

For the forward path, the least square method is used to identify the consequent parameters. Now, for a given set of values of elements of $P_{1}$, we can obtain the following matrix equation based on the train data:

$$
B X=Y \text {, }
$$

where $B$ contains the unknown parameters in $P_{2}, X$ is the input matrix, and $Y$ is the output.

A least squares estimate is sought to minimize $\|B X-Y\|^{2}$, which is a problem for linear regression. Thus, the calculation for the parameters set $B$ is:

$$
B^{*}=\left(X^{T} X\right)^{-1} X^{T} Y \text {. }
$$

In the backward path, the error signals propagate backward. The antecedent parameters are updated by the descent method, through minimizing the error between the output of the network and that of real system, which is a quadratic function:

$$
E(\Psi)=\frac{1}{2} \sum_{d=1}^{N}\left(y(d)-i_{f}(d)\right)^{2}
$$

where $\Psi$ represents the training data set and $N$ is the number of the training data.

In a recursive manner, with respect to $\Psi$, the update of the parameters of membership function can be written as

$$
\begin{gathered}
c_{A_{L}}(z+1)=c_{A_{L}}(z)-\eta \frac{\partial E}{\partial c_{A_{L}}} \\
\sigma_{A_{L}}(z+1)=\sigma_{A_{L}}(z)-\eta \frac{\partial E}{\partial \sigma_{A_{L}}},
\end{gathered}
$$

where $c_{A_{L}}$ and $\sigma_{A_{L}}$ are the centers of the function and the widths of the membership function. $\eta$ is the learning rate and $\eta>0$.

Therefore, the amplitude of fundamental wave current could be predicted correctly by the proposed algorithm and the harmonic elimination of EAF would be realized. In the next section, the experiments results will show the effectiveness of the proposed algorithm.

\section{Experiments Results}

In the section, some experiments are performed to evaluate the effectiveness of the proposed algorithm. The experiments focus on comparing the proposed algorithm with BPNN for estimating the fundamental wave amplitude and the field data are used in the experiments. The field data are collected at an EAF at the second side of furnace transformer and the sampling rate is $50 \mathrm{kHz}$ (1000 amples/per cycle for the ideal case of $f=50 \mathrm{~Hz}$ ). To facilitate analysis, the values of field data are normalized. For the experiments, the 4000 samples from field data are divided into the training set and the test set based on the Monte Carlo cross-validation at the ratio that equaled $4: 1$. The root mean squares error of prediction 
TABLE 1: RMSEP values of 10 times experiments for 10 inputs.

\begin{tabular}{lcc}
\hline Number & The proposed algorithm & BPNN \\
\hline 1 & $5.73 E-05$ & $2.6432 E-04$ \\
2 & $4.54 E-05$ & $1.7707 E-04$ \\
3 & $5.81 E-05$ & $1.2414 E-04$ \\
4 & $4.94 E-05$ & $1.5082 E-04$ \\
5 & $4.08 E-05$ & $1.6229 E-04$ \\
6 & $3.26 E-05$ & $1.4230 E-04$ \\
7 & $4.34 E-05$ & $1.7170 E-04$ \\
8 & $5.53 E-05$ & $1.8885 E-04$ \\
9 & $3.47 E-05$ & $2.4323 E-04$ \\
10 & $7.08 E-05$ & $2.2032 E-04$ \\
The average RMSEP & $\mathbf{4 . 8 7 6 4 E}-\mathbf{0 5}$ & $\mathbf{1 . 8 4 5 0 E}-\mathbf{0 4}$ \\
\hline
\end{tabular}

(RMSEP) is used to estimate the predictive ability of the proposed algorithm and BPNN. For the proposed algorithm, the training epoch number is 100 , the training error goal is zero, the initial step size is 0.01 , the step size decrease rate is 0.7 , and the step size increase rate is 1.3. For BPNN, the number of training epochs is 100 , the learning rate is 0.01 , the number of hidden neurons is 10 , and the training error goal is zero. Moreover, the measured amplitudes of fundamental waves of field data are obtained by SW-DFT on the field data. Because the inputs are the average of measured value in different time intervals, the number of inputs may not be fixed and could be set according to the analysis demand. Hence, the experiments are performed with different numbers of inputs and the results are discussed in the following.

For the number of inputs is 10, the RMSEP values of 10 times experiments are shown in Table 1. Since assessing the sampling bias, the division of field data and the calculation process would be implemented repeatedly. The average RMSEP values of the proposed algorithm and BPNN are $4.8764 E-05$ and $1.8450 E-04$, respectively. The proposed algorithm is more effective and improves the prediction precision by $73.57 \%$ than BPNN. According to the average RMSEP value, the measured value curve and predictions curves of the proposed algorithm and BPNN for other 2000 samples from field data are shown in Figure 5, and the models of the proposed algorithm and BPNN are based on the number 2 experiment results. It also shows that the proposed algorithm has higher effectiveness.

For the number of inputs is 20, the RMSEP values of 10 times experiments are shown in Table 2. The average RMSEP values of the proposed algorithm and BPNN are 3.92E - 06 and $6.77 E-05$, respectively. The proposed algorithm is more effective and improves the prediction precision by $94.21 \%$ than BPNN. According to the average RMSEP value, the measured value curve and predictions curves of the proposed algorithm and BPNN for the 2000 samples used in Figure 4 are shown in Figure 6, and the models of the proposed algorithm and BPNN are based on the number 9 experiment results. The predictive ability of BPNN is decreased with the number of inputs being increased and that of the proposed algorithm is still better. It also shows that the proposed algorithm has higher effectiveness.
TABLE 2: RMSEP values of 10 times experiments for 20 inputs.

\begin{tabular}{lcc}
\hline Number & The proposed algorithm & BPNN \\
\hline 1 & $3.92 E-06$ & $6.77 E-05$ \\
2 & $3.73 E-06$ & $7.23 E-05$ \\
3 & $3.72 E-06$ & $3.73 E-05$ \\
4 & $3.60 E-06$ & $4.12 E-05$ \\
5 & $3.63 E-06$ & $9.32 E-05$ \\
6 & $3.45 E-06$ & $2.59 E-05$ \\
7 & $3.45 E-06$ & $2.17 E-05$ \\
8 & $3.50 E-06$ & $9.61 E-05$ \\
9 & $3.68 E-06$ & $4.65 E-05$ \\
10 & $3.47 E-06$ & $9.52 E-05$ \\
The average RMSEP & $\mathbf{3 . 9 2 E}-\mathbf{0 6}$ & $\mathbf{6 . 7 7 E}-\mathbf{0 5}$ \\
\hline
\end{tabular}

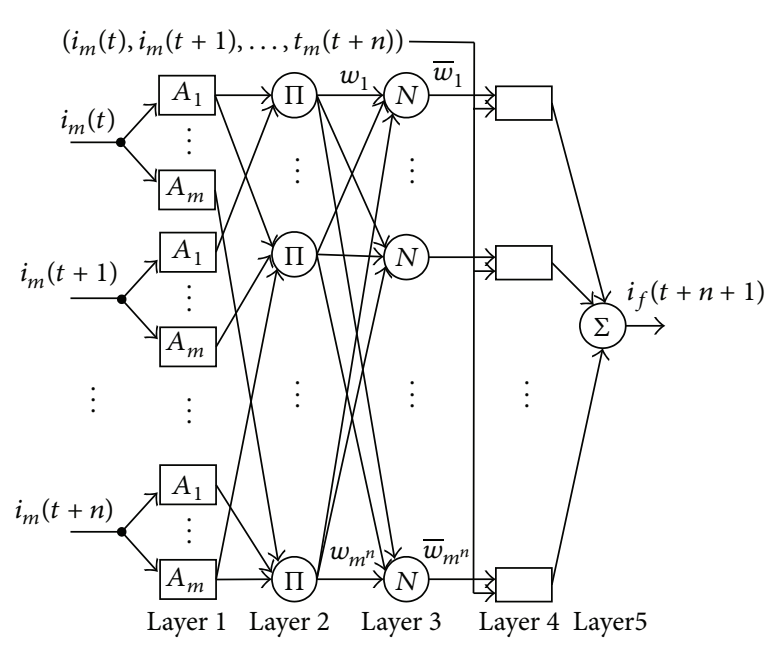

FIGURE 4: The structure of ANFIS used in the proposed algorithm.

Therefore, the experiments results verify that the effectiveness of the proposed algorithm is higher; namely, the amplitude of fundamental wave current could be predicted accurately, and the harmonic elimination of EAF would be realized based on the proposed algorithm.

\section{Conclusions}

The paper proposes a fundamental wave amplitude prediction algorithm based on FNN for harmonic elimination of EAF current. The proposed model has some advantages as follows. First, it has higher prediction capability for fundamental wave amplitude. Second, it is almost not affected by the number of inputs changed. Third, it uses the learning ability of the neural network to implement and refine Takagi-Sugeno type fuzzy rules to predict the fundamental wave amplitude. Fourth, by the hybrid learning process, all the best parameters of membership functions are obtained. The experiments results also verify the effectiveness of the proposed algorithm. Since the train time may be affected by the number of inputs, in the future research work, we will 


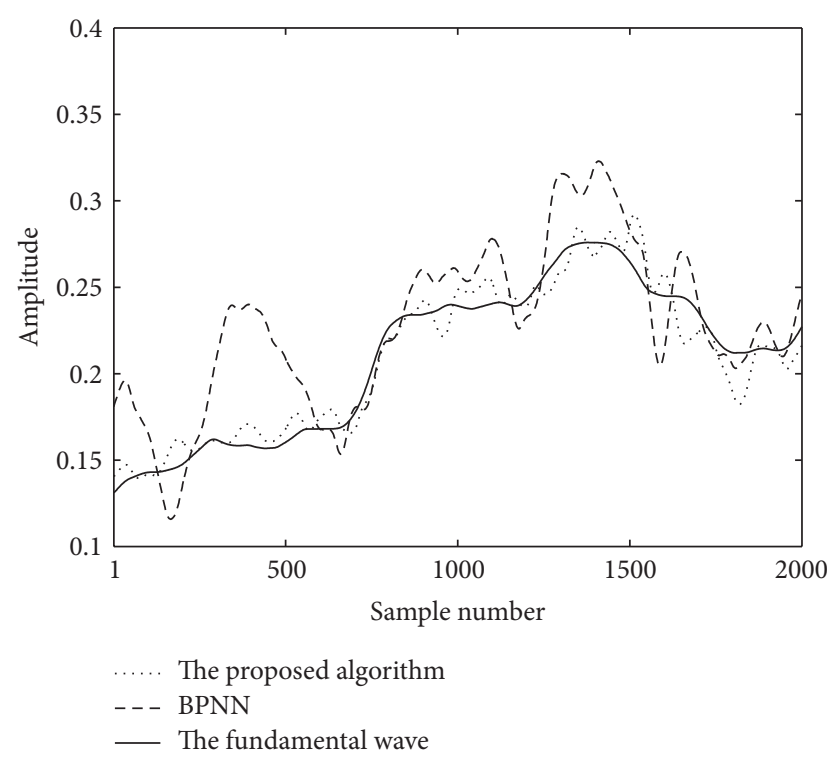

FIGURE 5: Experiments results for 10 inputs.

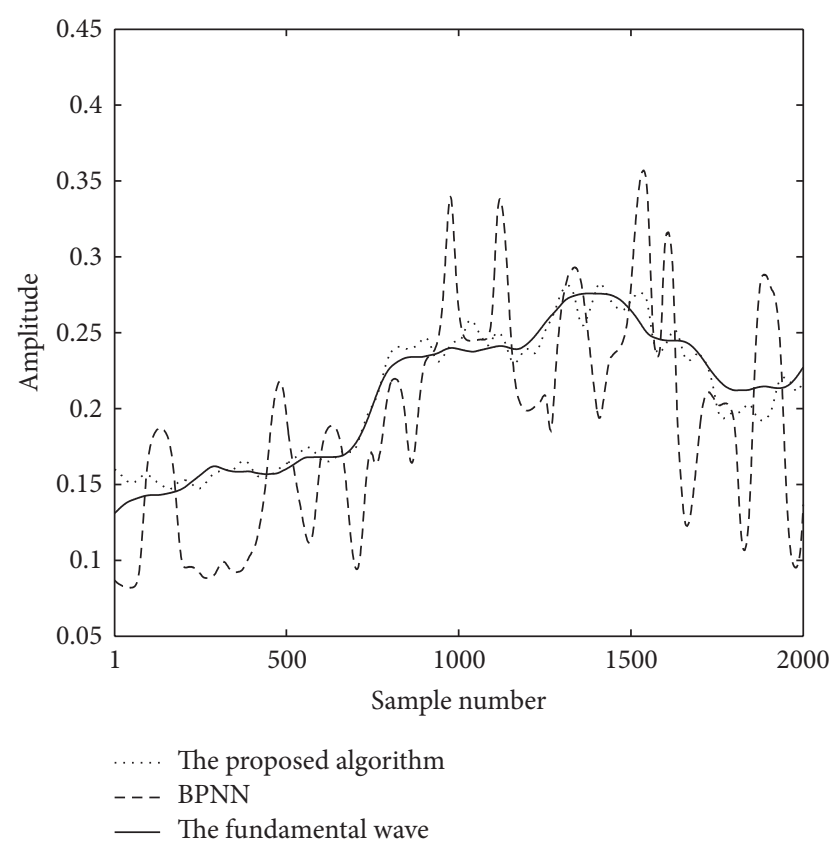

Figure 6: Experiments results for 20 inputs.

use some feature extraction approaches to further improve the training performance of the proposed algorithm.

\section{Conflict of Interests}

The authors declare that there is no conflict of interests regarding the publication of this paper.

\section{Acknowledgments}

This research was supported by the National Natural Science Foundation of China (51207126), the Delta Science \& Technology Educational Development Program (DREG2011009), and the Fundamental Research Funds for the Central University.

\section{References}

[1] A. H. Rahmat and T. E. Mahdi, "Adaptive filter design based on the LMS algorithm for delay elimination in TCR-FC compensators," ISA Transactions, vol. 50, no. 2, pp. 142-149, 2011.

[2] Y.-J. Liu, G. W. Chang, and R.-C. Hong, "Curve-fitting-based method for modeling voltage-current characteristic of an ac electric arc furnace," Electric Power Systems Research, vol. 80, no. 5, pp. 572-581, 2010.

[3] M. Göl, Ö. Salor, B. Alboyací, B. Mutluer, I. Çadírcí, and M. Ermis, "A new field-data-based EAF model for power quality studies," IEEE Transactions on Industry Applications, vol. 46, no. 3, pp. 1230-1242, 2010.

[4] V. Logar, D. Dovžan, and I. Škrjanc, "Mathematical modeling and experimental validation of an electric arc furnace," ISIJ International, vol. 51, no. 3, pp. 382-391, 2011.

[5] R. A. Hooshmand and S. Noohi, "Neural network-based algorithm for harmonic analysis of industrial systems using asynchronous simulations in time and frequency domains," IEEJ Transactions on Electrical and Electronic Engineering, vol. 5, no. 6, pp. 688-694, 2010.

[6] G. W. Chang, C.-I. Chen, and Y.-F. Teng, "Radial-basisfunction-based neural network for harmonic detection," IEEE Transactions on Industrial Electronics, vol. 57, no. 6, pp. 21712179, 2010.

[7] Y. Han, L. Xu, M. M. Khan, G. Yao, L.-D. Zhou, and C. Chen, "Study on a novel approach to active power filter control using neural network-based harmonic identification scheme," Electrical Engineering, vol. 91, no. 6, pp. 313-325, 2010.

[8] H. Temurtas and F. Temurtas, "An application of neural networks for harmonic coefficients and relative phase shifts detection," Expert Systems with Applications, vol. 38, no. 4, pp. 3446-3450, 2011.

[9] A. Ketabi, I. Sadeghkhani, and R. Feuillet, "Using artificial neural network to analyze harmonic overvoltages during power system restoration," European Transactions on Electrical Power, vol. 21, no. 7, pp. 1941-1953, 2011.

[10] F. Filho, H. Z. Maia, T. H. A. Mateus, B. Ozpineci, L. M. Tolbert, and J. O. P. Pinto, "Adaptive selective harmonic minimization based on ANNs for cascade multilevel inverters with varying DC sources," IEEE Transactions on Industrial Electronics, vol. 60, no. 5, pp. 1955-1962, 2013.

[11] A. Guellal, C. Larbes, D. Bendib, L. Hassaine, and A. Malek, "FPGA based on-line artificial neural network selective harmonic elimination pwm technique," International Journal of Electrical Power \& Energy Systems, vol. 68, pp. 33-43, 2015.

[12] H. Khoshkhoo, S. H. H. Sadeghi, R. Moini, and H. A. Talebi, "An efficient power control scheme for electric arc furnaces using online estimation of flexible cable inductance," Computers and Mathematics with Applications, vol. 62, no. 12, pp. 4391-4401, 2011.

[13] J.-S. R. Jang, "ANFIS: adaptive-network-based fuzzy inference system," IEEE Transactions on Systems, Man and Cybernetics, vol. 23, no. 3, pp. 665-685, 1993. 
[14] B. M. Dehkordi, M. Moallem, and A. Parsapour, "Predicting foaming slag quality in electric arc furnace using power quality indices and fuzzy method," IEEE Transactions on Instrumentation and Measurement, vol. 60, no. 12, pp. 3845-3852, 2011.

[15] K. C. Lee and P. Gardner, "Adaptive neuro-fuzzy inference system (ANFIS) digital predistorter for RF power amplifier linearization," IEEE Transactions on Vehicular Technology, vol. 55, no. 1, pp. 43-51, 2006. 


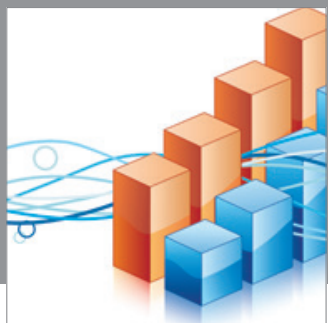

Advances in

Operations Research

mansans

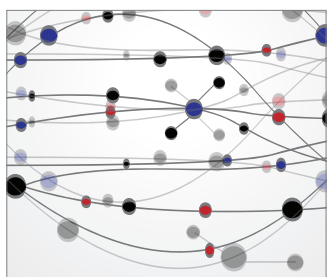

The Scientific World Journal
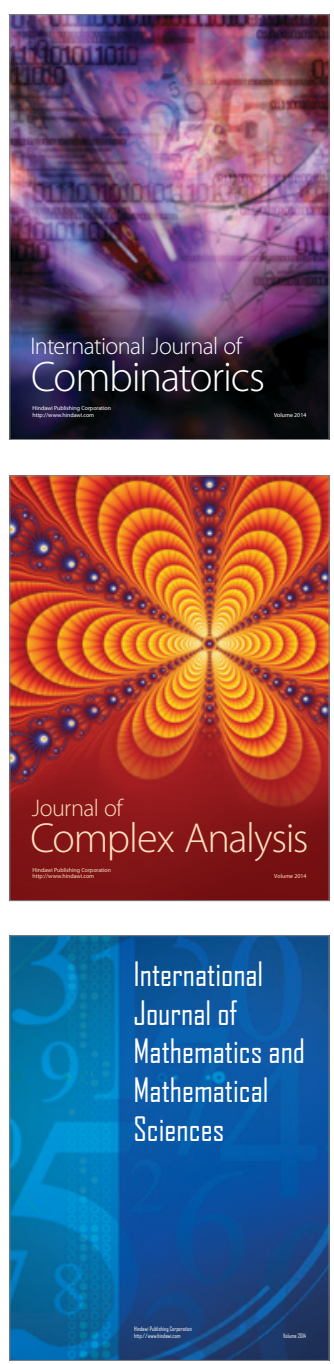
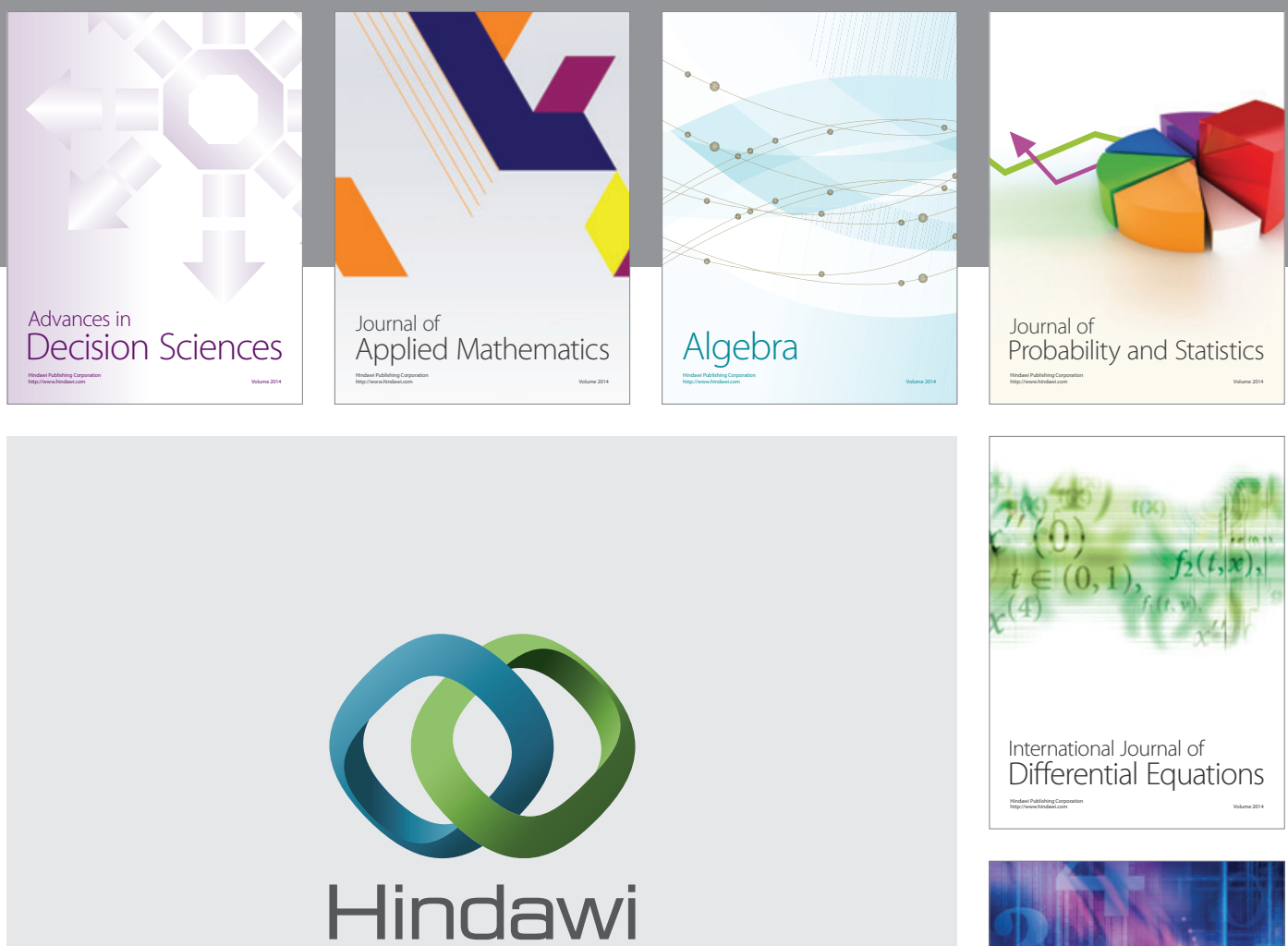

Submit your manuscripts at http://www.hindawi.com
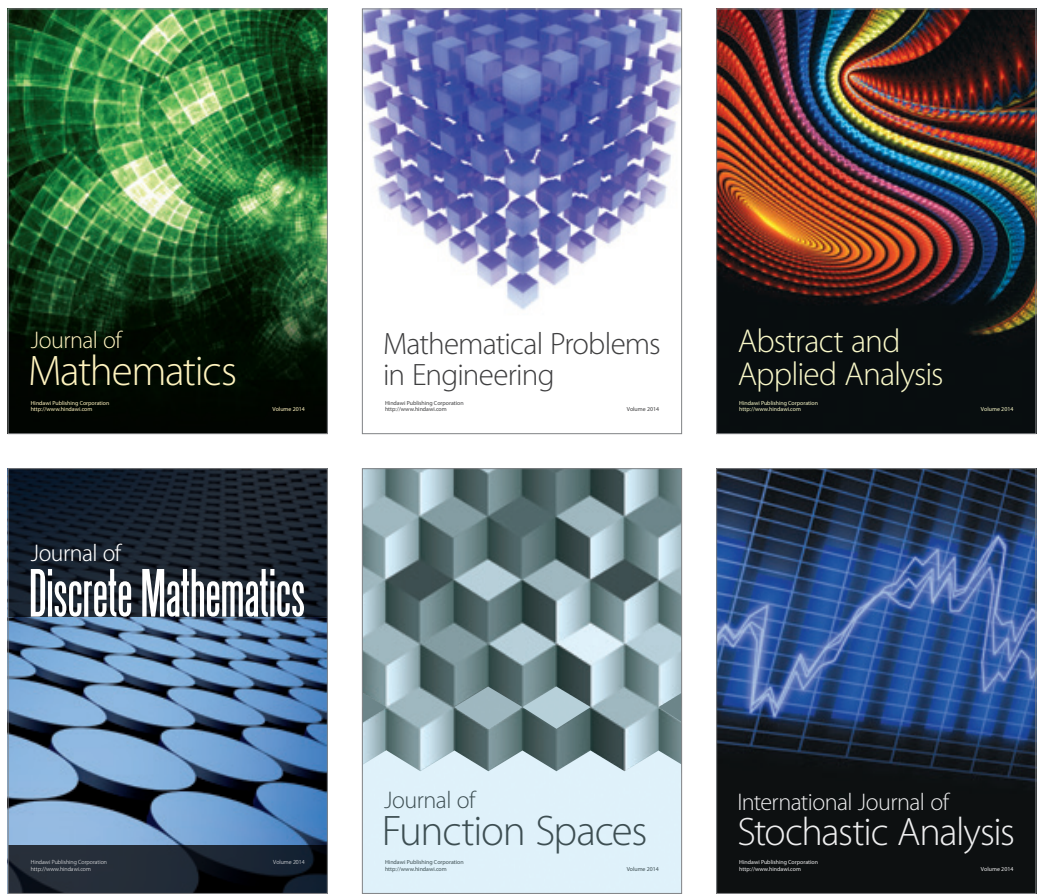

Journal of

Function Spaces

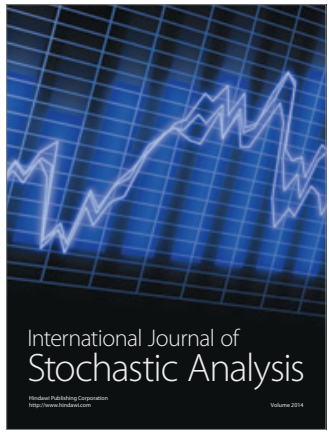

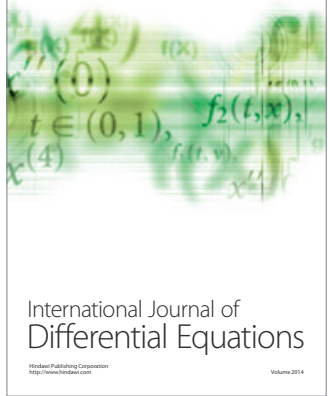
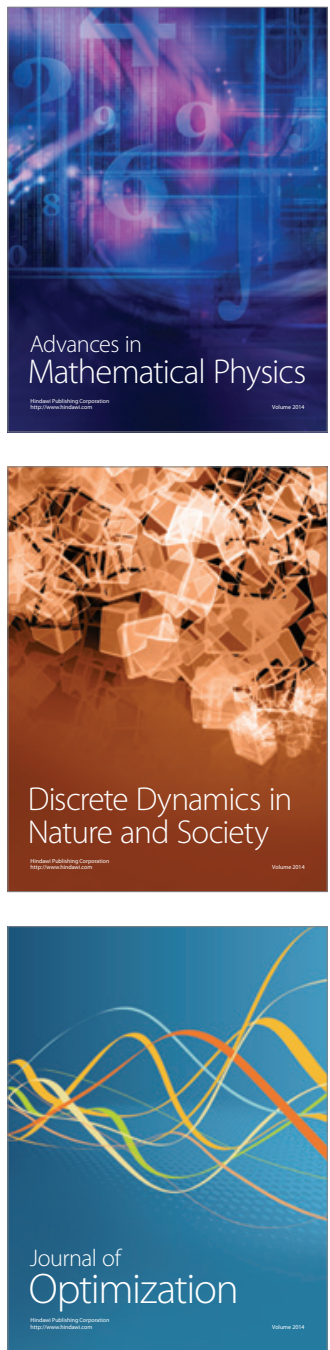